\author{
Shoko Yoshida \\ Katsumi Hayakawa \\ Akira Yamamoto \\ Hiroshi Kuroda \\ Shinsaku Imashuku
}

\section{The central nervous system complications of bone marrow transplantation in children}

Received: 6 January 2008

Revised: 24 March 2008

Accepted: 29 March 2008

Published online: 20 May 2008

(C) The Author(s) 2008
S. Yoshida $(\bowtie) \cdot$ K. Hayakawa

A. Yamamoto

Department of Radiology,

Kyoto City Hospital,

1-2 Higashi Takada-cho, Mibu,

Nakagyo-ku,

Kyoto, 604-8845, Japan

e-mail: sho-ko@mx.biwa.ne.jp

Tel.: +81-75-3115311

Fax: $+81-75-3216025$

H. Kuroda $\cdot$ S. Imashuku

Department of Pediatrics,

Kyoto City Hospital,

Kyoto, Japan

\author{
Abstract Bone marrow transplanta- \\ tion (BMT) is widely performed for \\ both neoplastic and non-neoplastic \\ disease. Before BMT, patients are \\ prepared with high-dose chemo- \\ therapy, frequently associated with \\ total-body radiation, to destroy \\ residual malignant cells and to reduce \\ immunologic resistance. BMT is \\ associated with several central ner- \\ vous system (CNS) complications \\ secondary to underlying disease, pro- \\ longed myelosuppression, and the use \\ of immunosuppressive drugs. These \\ complications include infections, \\ vascular disease, drug-induced neuro- \\ toxicity, metabolic disturbance, and \\ post-BMT carcinogenesis. The \\ immune status of children after BMT \\ can be divided into three phases: the \\ pre-engraftment period (days 0-30 \\ after BMT), the post-engraftment
}

period (days 30-100), and the late phase (after day 100). The timing of CNS complications that occur after BMT, as for complications in other organs, can be described with reference to these three phases of immune status. It is essential that radiologists become familiar with the relationships between the immune status of the recipient and the times of onset of these disorders, and with the neuroimaging patterns associated with the various complications. CNS complications can be life-threatening for immunosuppressed children, so accurate diagnosis is important for prompt and appropriate treatment.

Keywords Bone marrow transplantation - Central nervous system · Immunosuppression · Pediatrics

\section{Introduction}

Bone marrow transplantation (BMT) is performed for the treatment of pediatric patients with hematologic malignancies (leukemia, malignant lymphoma), solid neoplasms, bone marrow aplasia, and a variety of congenital dyshematopoietic and metabolic diseases [1-3]. The particular approaches used have a variety of names depending on the relationship between the marrow donor and the recipient and on the source of the blood stem cell (Table 1) [1-3]. In this review we consider all types of donors and recipients and use bone marrow transplantation (BMT) as an inclusive term.
Before BMT, the patient is prepared with high doses of chemotherapy, frequently associated with total-body irradiation, to destroy the hematopoietic system and to suppress the immune system, especially T-cells, so that graft rejection is prevented. In patients with malignancies, the preparative regimen also serves to reduce the tumor burden. These preparative therapies can predispose patients to infection, thrombocytopenia, metabolic disturbance, and potential neurotoxicity, including impaired development of the nervous system. Among these complications, the frequency of central nervous system (CNS) involvement associated with BMT is reported to be $11-65 \%(90 \%$ at autopsy) of BMT recipients, and these CNS complications 


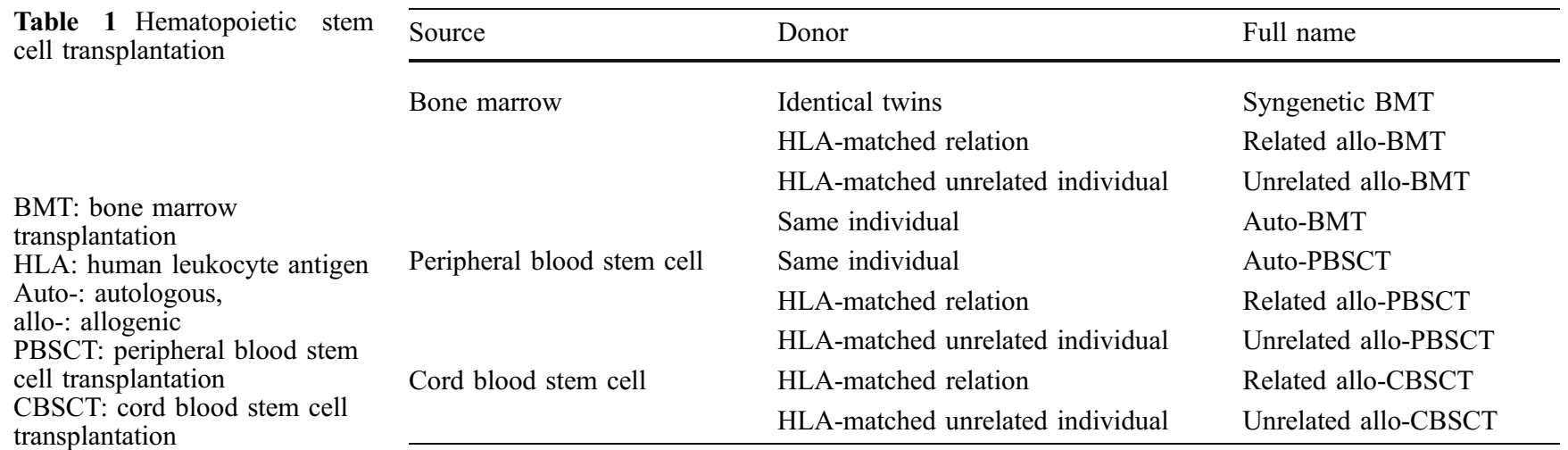

may be the main causes of death in 9-17\% [4-10]. To begin proper treatment as early as possible, accurate diagnosis is important and imaging [magnetic resonance imaging (MRI) and computed tomography (CT)] is helpful for diagnosis.

In this paper, we review the characteristic imaging findings and appearances of CNS complications associated with BMT in children and correlate these complications with clinical background according to the time elapsed after BMT.

\section{Immune status following BMT}

BMT recipients experience a transient, but profound state of immune deficiency. The immune status of children after BMT can be divided into three phases: the pre-engraftment period (phase I, days 0-30 after BMT), the postengraftment period (phase II, days 30-100), and the late phase (phase III, after day 100) (Table 2) [1,9,11]. During phase I, the pre-engraftment period ( $<$ day 30$)$, the immune system of patients is completely suppressed and the hemopoietic system is destroyed as a result of the preparative conditioning regimen for BMT, which involves high-dose chemotherapy often associated with irradiation. Pancytopenia may occur, especially associated with severe neutropenia (less than $100 / \mu \mathrm{l}$ ), causing a high risk of infection. In the next phase, the post-engraftment period (days 30-100), engraftment of donor marrow is confirmed, and immune recovery starts, although immune depletion (cellular immunodeficiency) continues. Acute graft versus host disease (GVHD) may develop, caused by alloreactive donor-derived T-cells in the graft attacking nonshared recipient antigens in target tissues (skin, liver, and intestinal tract), resulting in erythematous maculopapular rash, persistent anorexia, vomiting and/or diarrhea, and liver dysfunction. The high dose of chemotherapy associated with irradiation during the conditioning regimen before BMT can cause damage in host tissues including the mucosa, liver, and other tissues. Activated cells from damaged recipient tissues secrete many inflammatory cytokines, such as interleukin-1 (IL-1), tumor necrosis factor (TNF)-alpha, granulocyte-macrophage colony-stimulating factor (GM-CSF), and interferon (IFN)-gamma, which may upregulate expression of recipient antigens. After BMT (commonly during phase II, days 30-100), presentation of recipient antigens to donor T cells occurs, and subsequent proliferation and differentiation of these activated $\mathrm{T}$ cells is crucial in this phase of GVHD. Activated CD4+ T-cells (helper $\mathrm{T}$ cells) expand and generate cytokines such as tumor necrosis factor (TNF)alpha, interleukin-2, and interferon-gamma. These cytokines cause tissue damage and promote differentiation of CD8+ T-cells to cytotoxic T-cells, which kill recipient cells
Table 2 Immune status after BMT

\begin{tabular}{|c|c|c|}
\hline $\begin{array}{c}\text { Phase I: } \\
\text { pre-engraftment period } \\
(<\text { day } 30)\end{array}$ & $\begin{array}{c}\text { Phase II: post-engraftment } \\
\text { period (day } 30-100)\end{array}$ & $\begin{array}{c}\text { Phase III: late phase } \\
\text { (> day 100) }\end{array}$ \\
\hline $\begin{array}{l}\text { - Severe bone marrow } \\
\text { suppression } \\
\text { - Neutropenia }(<100 / \mu \mathrm{l}) \\
\text { - Damage to mucosal } \\
\text { barrier }\end{array}$ & $\begin{array}{l}\cdot \text { Acute GVHD } \\
\text { - Continuous immune } \\
\text { depletion (Cellular } \\
\text { immunodeficiency) }\end{array}$ & $\begin{array}{l}\cdot \text { Chronic GVHD } \\
\text { - Progressive immune } \\
\text { functions recovery } \\
\text { (residual cellular and } \\
\text { humoral } \\
\text { immunodeficiency) }\end{array}$ \\
\hline
\end{tabular}


and further disrupt tissues. The prophylaxis of GVHD mainly consists of post-transplant administration of immunosuppressive drugs such as cyclosporine or tacrolimus, with methotrexate, steroids, anti T-cell antibodies, and other immunosuppressive agents. These GVHD prophylaxes can impair post-transplant immunologic reconstitution. After about 100 days (late phase), immune recovery occurs progressively towards complete recovery. Chronic GVHD may develop and is the most frequent late complication. In chronic GVHD, patients with only liver and skin involvement have a favorable prognosis. By comparison, patients with chronic GVHD and extensive involvement of multiple organ systems may experience prolonged cellular and humoral immunosuppression associated with the immunosuppressive regimens necessary to control GVHD, and the prognosis may be poor. Graft failure or relapse of underlying disease can occur during this phase $[1,11]$.

The timing of CNS complications that occur after transplantation is similar to the timing of complications in other organs and can be described with reference to the three phases of recipient immune status (Table 3) [1,2,11, 12]. It is very important for radiologists to appreciate how the different timing of onset of complications and their neuroimaging patterns can vary according to the immune status of the patient. Infections, vascular disease, druginduced neurotoxicity, metabolic disturbance, and postBMT carcinogenesis are the important CNS complications after BMT in children.

\section{Infectious disease}

Infectious disease is the most common CNS complication of BMT (2.2-14\%) and one of the most common causes of morbidity after BMT, although the incidence has reduced recently. Susceptibility to different infectious agents varies with the nature of immune impairment after BMT [4-13].

During the first phase $(<$ day 30$)$, there is a particularly high risk of certain fungal infections (Aspergillus, Candi$d a$ ), gram-negative bacterial infections and viral infections, although prophylactic antibiotic or antifungal treatment is commonly a component of conditioning regimens (Fig. 1). During the second period (days 30-100), which is characterized by profound deficiency of cellular immunity, GVHD is itself an additional risk factor for fungal and viral opportunistic infections, as are the immunosuppressive agents. CNS involvement is predominantly with virus (human herpesvirus-6, cytomegalovirus), fungi, Toxoplas$m a$, and gram-positive bacteria. In the late phase ( $>$ day 100 ), infections caused by encapsulated bacteria and herpes zoster virus are commonly seen, and progressive multifocal leukoencephalopathy (PML) may rarely occur [11].

Aspergillosis

Aspergillosis is the most common CNS infection after BMT, especially during the first and second periods. In
Table 3 CNS complications after BMT

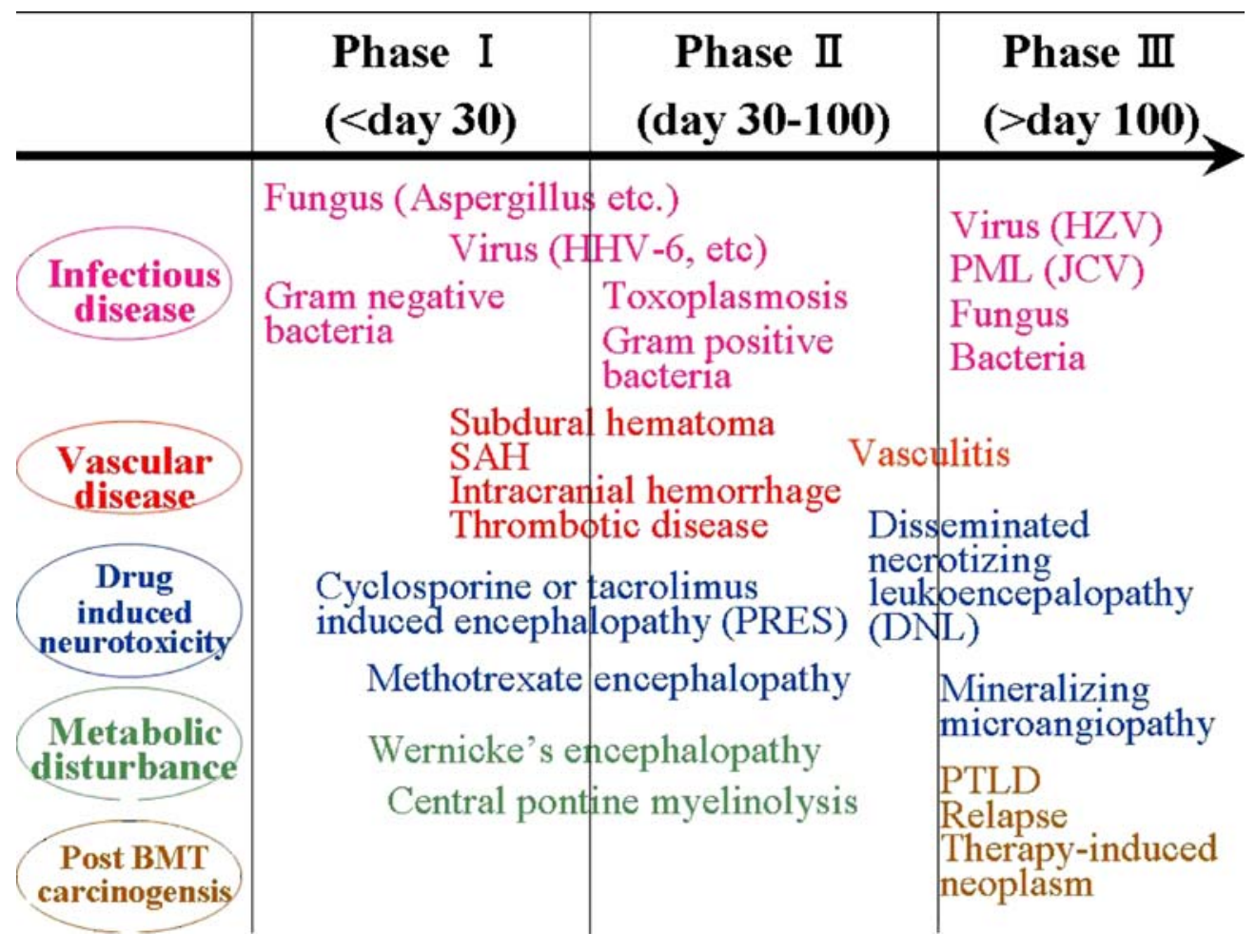




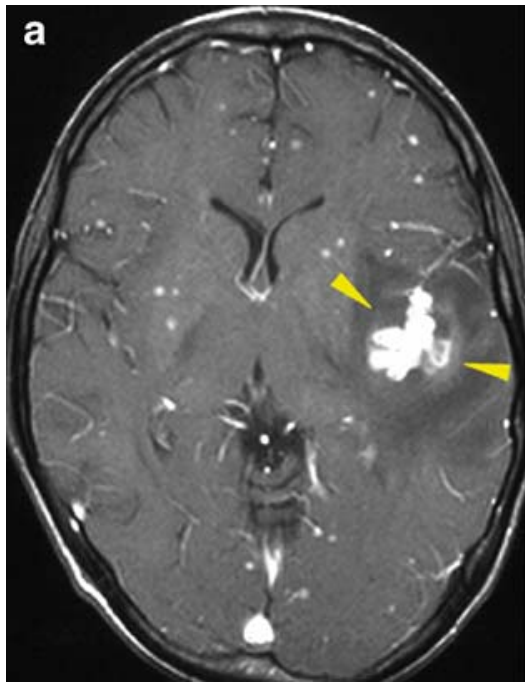

Fig. 1 A 17-year-old boy after BMT 4 years ago due to hepatic undifferentiated sarcoma presented with acute myeloblastic leukemia. Chemotherapy with PBSC rescue was performed. The next day, he had fever, cognitive and memory disturbance, and ataxia. Axial FLAIR magnetic resonance (MR) image shows abnormal hyperintense signal in the left temporal lobe with multiple spotty hyperintense lesions dominantly in subcortical white matter (not shown). (a) Post-contrast T1-weighted axial image shows the lesion

most cases, invasive aspergillosis develops in the paranasal sinuses and in the lungs, and spreads secondarily to the brain. Involvement of the spinal cord is very rare. The mortality rate for cerebral aspergillosis approaches 85$100 \%$, depending on the degree of neurological compromise at presentation [14-15]. Presenting symptoms are nonspecific (stroke-like symptoms or seizures), and fever may be absent. The organism is rarely cultured from cerebrospinal fluid, so the clinical diagnosis of cerebral aspergillosis can be difficult.

Imaging findings Vasculopathy and secondary multiple septic infarcts occur, involving the lenticulostriate and thalamoperforating artery distributions, often associated with hemorrhage and abscess formation. Involvements of basal ganglia, thalami, and the corticomedullary area are common. Brain-computed tomography (CT) shows multiple irregular, low attenuated areas with little enhancement after administration of intravenous contrast medium, often associated with high attenuation due to hemorrhage (Fig. 2). Magnetic resonance imaging (MRI) detects many more lesions than CT. On T2-weighted image (T2WI), intermediate-low intensity within a surrounding high signal area can be seen. Diffusion-weighted images are useful for early detection of multiple septic infarcts. Ringlike enhancement is often absent, reflecting the deficiency of inflammatory response caused by the severely immunosuppressed status. In a less severely immunocompromised status, there may be ring-like enhancement. T2WI and FLAIR images are also useful for detection of

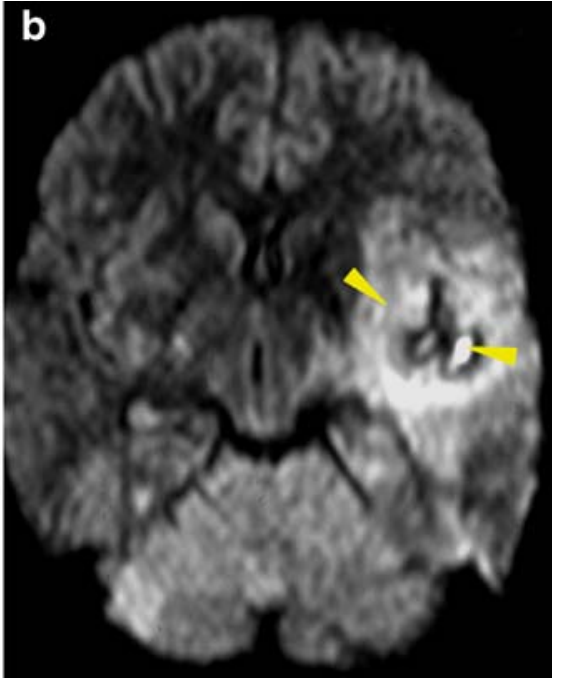

enhanced and partially ring-like enhanced in the left temporal lobe (arrowhead). (b) Axial diffusion-weighted image and ADC map (not shown) reveal diffusion restriction of the lesion in the left temporal lobe (arrowhead). Fungal infection with microabscess formation was suspected. Antifungal therapy was performed, and his neurological symptoms improved and the abnormal small nodules with microabscesses disappeared

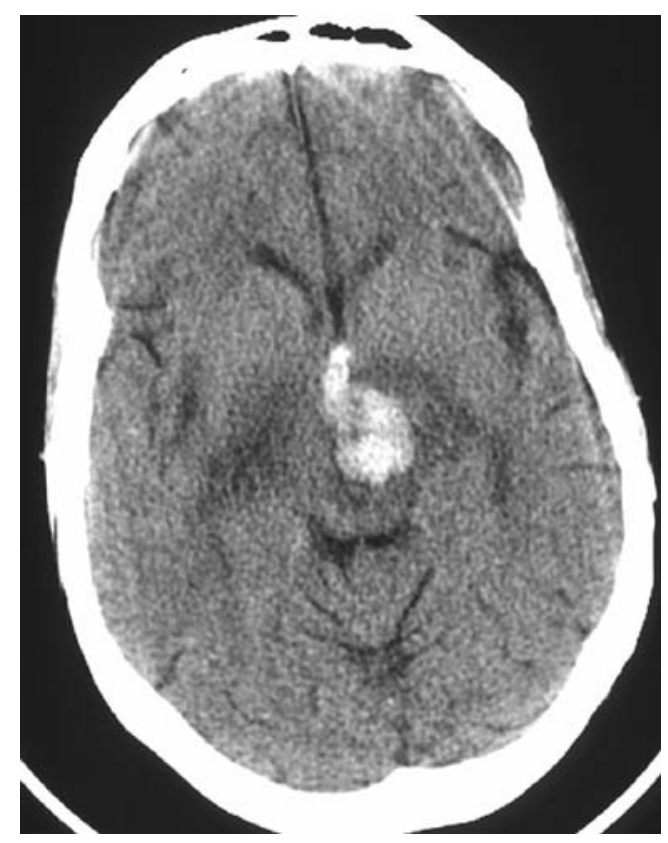

Fig. 2 A 25-year-old man after BMT because of Hodgkin disease and secondary acute myeloid leukemia, complicated by acute GVHD. He experienced sudden onset of left pupil dilatation and left hemiparesis. Plain axial CT images revealed a low-attenuated lesion in the right internal capsule and right frontal lobe, associated with hemorrhage (not shown). Follow-up CT obtained 2 days later showed hemorrhage of the midbrain lesion with perforation to the third ventricle. The patient died $8 \mathrm{~h}$ after the second CT study. Autopsy revealed evidence of systemic aspergillosis (courtesy of Dr. K. Yamada, Kyoto Prefectural University of Medicine) 
additional lesions containing hyphae without an inflammatory reaction in an immunocompromised host; these appear as small, subcortical hyperintense lesions in the subcortical area with no contrast enhancement [14-17].

Human herpes virus-6 (HHV-6) encephalitis

In most children, primary HHV-6 infection occurs between 6 months and 5 years of age $[1,18,19]$. After the primary infection, HHV-6 probably remains latent in the body and then reactivates upon host immunosuppression, similarly to other human herpes viruses. Systemic reactivation of HHV6 develops in $28-78 \%$ of BMT recipients, usually in the first or second phase after BMT, and is associated with acute GVHD, although encephalitis occurs in only a minority [18-19]. Because of premedication with acyclovir or gancyclovir, cytomegalovirus (CMV), herpes simplex virus (HSV), and herpes zoster virus (HZV) infections are less common, although gancyclovir may cause leukopenia and T-cell immune suppression. The clinical symptoms of HHV-6 encephalitis are anterograde amnesia, change in mental state, headache, fever, and drowsiness. Polymerase chain reaction (PCR) detection of HHV-6 DNA in the cerebrospinal fluid in association with CNS symptoms has been shown to have a high sensitivity. The outcome may vary from near complete recovery to death, and the mortality is more than $50 \%$, although prompt treatment with foscarnet is dramatically effective [18-19].

Imaging findings Characteristic involvement of the limbic system is typical, including the medial temporal lobes, hippocampi, insula, subfrontal and cingulate gyri. Lesions are often hemorrhagic. CT findings in patients with HHV6 encephalitis are usually negative. MRI can also be normal in the first few days. Thereafter, T1 and T2 prolongation in the grey and subcortical white matter of the bilateral limbic systems can be detected, with contrast enhancement. Axial and coronal T2WI and FLAIR images have high sensitivity in the early detection of HHV-6 encephalitis as symmetrical abnormal hyperintensity in the limbic system. On diffusion-weighted images, these lesions are shown as symmetrical hyperintensity with a low apparent diffusion coefficient (ADC) in the early stage [18-21] (Fig. 3)

\section{Bacterial infection}

In the second phase (days 30-100), gram-positive bacterial infections are predominant, although they have decreased in frequency because of prophylactic usage of antibiotics $[1,11,12]$. Methicillin-resistant Staphylococcus aureus (MRSA), methicillin-resistant Staphylococcus epidermidis (MRSE), and Listeria monocytogenes may cause severe meningitis, encephalitis, and ventriculitis. The clinical symptoms are nonspecific: fever, headache, drowsiness, and seizures.

Imaging findings Lesions are more commonly detected with MRI than CT. In meningitis, abnormal thickening and enhancement of the leptomeninges are detectable on MRI with contrast enhancement. In pyogenic ventriculitis, hydrocephalus and abnormal enhancement of the ventricular lining with diffusion restriction are typical findings on MRI, with intraventricular debris and pus deposition, and occasionally abnormal thickening and enhancement of the choroid plexus (Fig. 4). Diffusion-weighted image (DWI) has higher sensitivity than any other sequence for detection of intraventricular and periventricular signal abnormality [22-25].

\section{Progressive multifocal leukoencephalopathy (PML)}

During the late phase (>day 100) after BMT, reactivation of the JC virus may develop in $10-15 \%$ of recipients with asymptomatic viruria. JC virus is tropic for renal epithe-
Fig. 3 A 22-year-old boy after BMT (day 19) due to non-

Hodgkin lymphoma associated with Langerhans cell histiocytosis. He experienced sudden onset of anterograde amnesia and seizure. a Coronal FLAIR image performed after 2 days shows hyperintense lesion in the medial temporal lobe clearly. b On axial diffusion-weighted image, bilateral medial temporal lobe is detected as symmetrical hyperintense lesion. HHV-6 DNA was detected in the CSF by using quantitative PCR
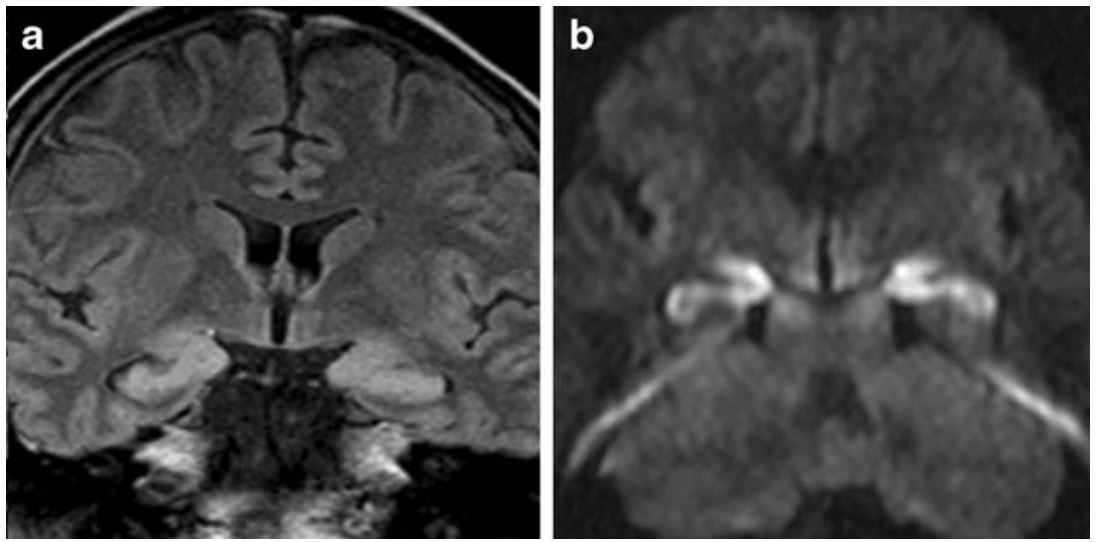


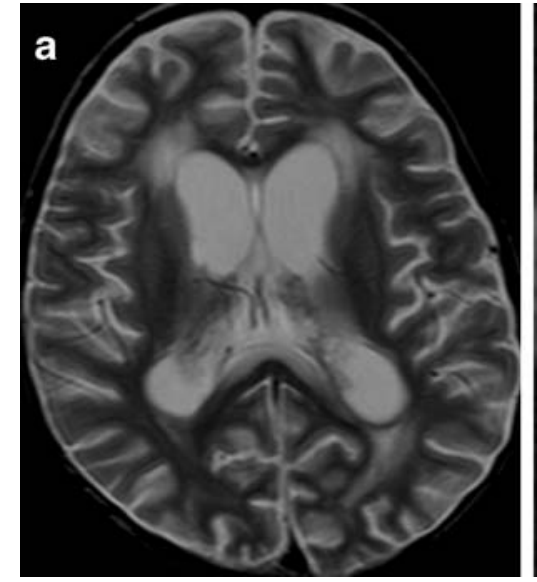

Fig. 4 A 3-year-old boy after BMT (day 77) because of myelodysplastic syndrome, complicated by GVHD. He had a fever and presented with a stiff neck. MRI was performed on day 78 after BMT. a Axial T2-weighted MR image shows ventriculomegaly with periventricular hyperintensity. Atrophy and hyperintensity of

lium and may infect oligodendrocytes in the brain, and is thus the etiologic agent of PML [1]. Pathologic features are multifocal demyelination with intranuclear inclusion bodies in oligodendrocytes. Unlike HIV infection or other organ transplantation, BMT rarely causes PML (only eight adult cases have been reported), and we could not find any report of pediatric PML after BMT. In adults, slowly progressive mental deterioration, sensory deficits, paralysis, ataxia, and visual disturbance are the clinical symptoms $[17,26]$.

Imaging findings Imaging findings of PML are similar in children and adults. On CT, multiple areas of decreased attenuation are typical. MRI shows multiple subcortical

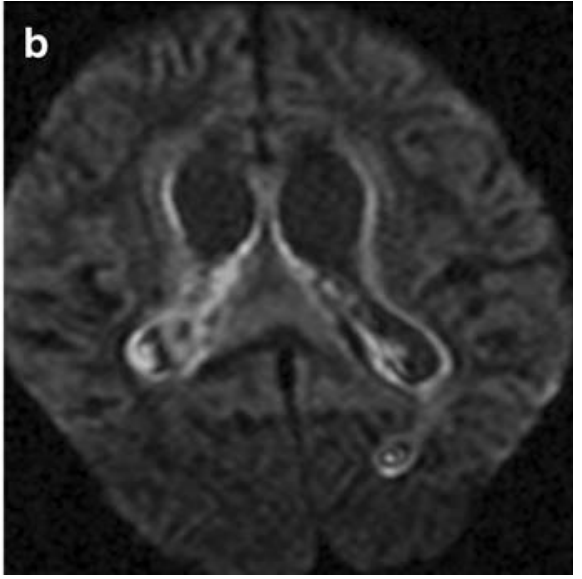

the white matter are also seen due to drug-induced neurotoxicity. b Axial diffusion-weighted image and ADC map (not shown) reveal the periventricular lesion with diffusion restriction. MRSA was detected in the cultured CSF

white matter lesions with $\mathrm{T} 1$ and $\mathrm{T} 2$ prolongation in the frontal and parieto-occipital regions (Fig. 5). The lesions lack mass effect and do not enhance with contrast material $[17,26]$.

\section{Vascular disease}

Cerebrovascular disease occurs in $3.8-8.8 \%$ of BMT recipients (more than $50 \%$ at autopsy), mostly during the first or second phases after BMT [4-9]. Subdural hemorrhage is most common, reflecting thrombocytopenia. Intraparenchymal hemorrhage, subarachnoid hemorrhage, and infarction may also develop (Fig. 6). Thrombotic
Fig. 5 Adult case of progressive multifocal leukoencephalopathy (PML). A 66-year-old woman treated for AML. a Axial T2-weighted MR image shows multiple diffuse or patchy lesions predominantly in the bilateral subcortical white matter presenting T2 prolongation. b On T2-weighted midsagittal MR image, involvement of the corpus callosum can be seen (courtesy of Dr. H. Tanaka, Osaka University)
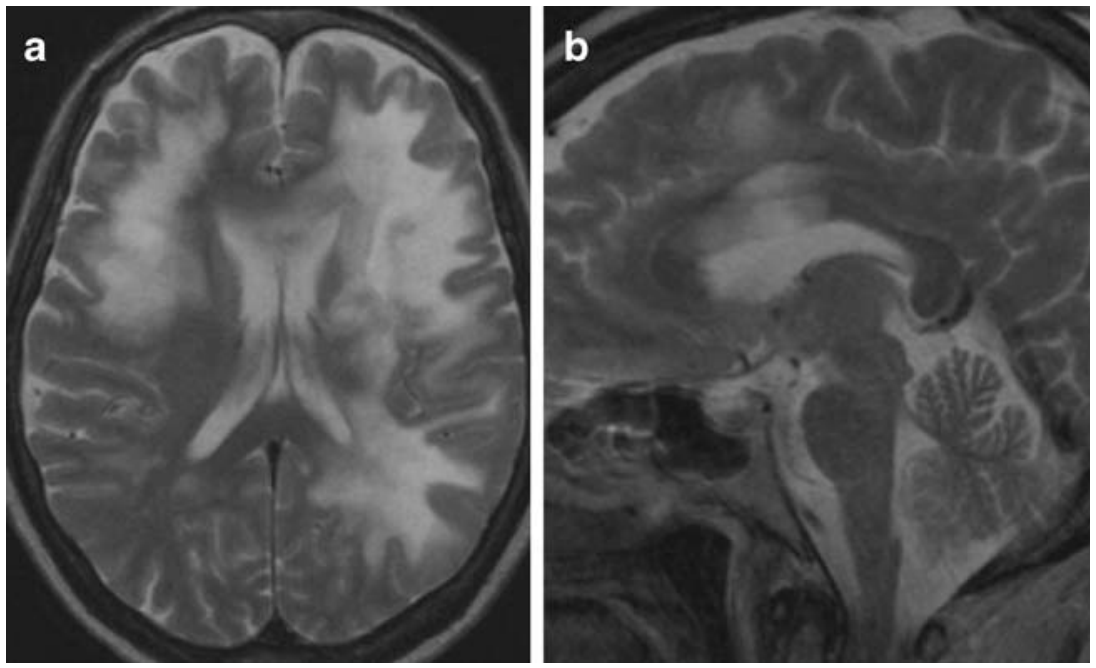


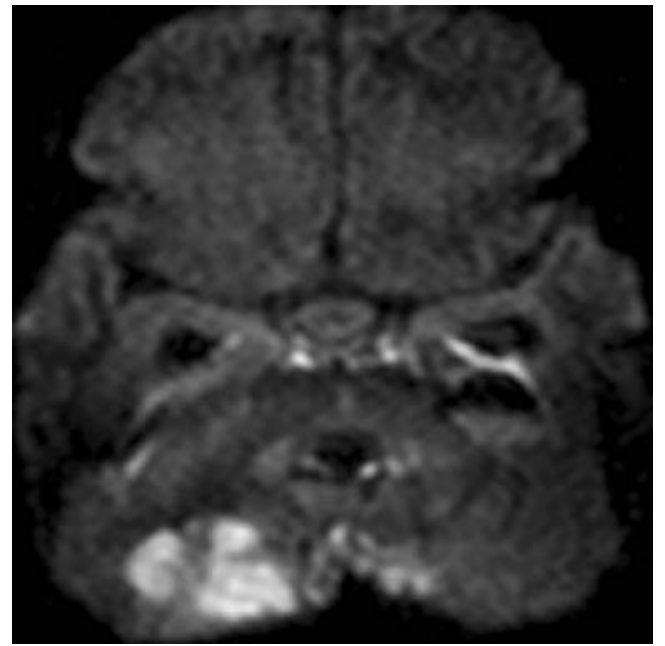

Fig. 6 A 3-year-old boy after BMT (day 87) for myelodysplastic syndrome. He was treated with immunosuppressive drugs and transfusion for GVHD and severe pancytopenia due to dyshematopoiesis. A plain axial CT scan reveals a low density lesion in the right cerebellar hemisphere (not shown). Axial diffusion-weighted image performed on the same day shows marked diffusion restriction in the right cerebellar lesion. Subacute cerebellar infarction was diagnosed

diseases are reported to be associated with endocarditis, indwelling catheter, and characteristically with treatment with L-asparaginase in ALL children [27-28]. During the second and late phases, CNS angiitis can occur with GVHD [29].

\section{Drug-induced neurotoxicity}

During the pre-transplantation period, high doses of chemotherapy, frequently associated with total-body irradiation, are administered to destroy residual malignant cells and to reduce immunologic resistance. In the post-transplantation period, immunosuppressive drugs such as methotrexate, cyclosporine, tacrolimus, and steroids are administrated as prophylaxes against GVHD. These drugs, with or without irradiation, can themselves induce CNS complications that may be life-threatening. MRI is generally the optimal imaging investigation in the followup. On MRI, drug-induced neurotoxicity generally presents with high signal intensity on T2WI and FLAIR and low signal intensity on T1WI, reflecting impairment of the blood-brain barrier due to endothelial damage and vasogenic edema, demyelination, gliosis, ischemia, and tissue necrosis [30].

From the time of prophylactic chemotherapy before BMT to a few weeks after BMT (first phase), an acute posttherapy syndrome may occur, commonly presenting as vasogenic edema resulting from damage to the capillary endothelium. From a few weeks to a few months after
BMT, an early delayed reaction may develop, including demyelination, astrogliosis, multifocal coagulative necrosis, and cavitation, transient in many cases. Disseminated necrotizing leukoencephalopathy has a poor neurological prognosis. During the late phase, from several months to several years after BMT, a late delayed reaction develops that reflects vascular changes with secondary ischemic changes causing tissue damage. Damage to the endothelium may lead to change in the vessel wall (e.g., resulting in moyamoya-like collaterals) [30].

\section{Posterior reversible encephalopathy syndrome (PRES)}

Immunosuppressive medications, such as cyclosporine-A, tacrolimus, and steroids, can induce PRES. The mechanism is still unclear, although the immunosuppressive drug itself may disrupt the blood-brain barrier or interfere with cerebrovascular autoregulation, resulting in vasogenic edema. PRES can potentially progress to irreversible cytotoxic edema [31-33].

Commonly, within 1 month of the beginning of prophylactic treatment (first and second phases), a usually reversible neurogenic syndrome develops with headache, altered mental status, seizures, and loss of vision or consciousness. The immunosuppressive and/or chemotherapeutic drugs causing PRES should be discontinued or switched. Once treatment is stopped, further deterioration may occur for 1 or 2 weeks before improvement. Treatment of hypertension, if any, is needed. Complete disappearance of clinical and imaging findings may occur, but residual sequelae are not rare [31-35].

Imaging findings $\mathrm{CT}$ shows hypodense lesions predominantly in the parieto-occipital regions. However, MRI is more sensitive than CT, and FLAIR appears to be the most sensitive pulse sequence. Typically, FLAIR images demonstrate hyperintensity in the parieto-occipital and posterior frontal cortical and subcortical white matter (Fig. 7). Less commonly, the brainstem, basal ganglia, and cerebellum are involved (Fig. 8). In general, the lesions are symmetric, but asymmetrical presentations are not rare. The brainstem and deep white matter lesions may tend to be irreversible. Atypical imaging findings include contrast enhancement, hemorrhage, and restricted diffusion on MRI, which are correlated with the extent of hyperintensity and mass effect on FLAIR [31-34].

\section{Methotrexate (MTX)-induced leukoencephalopathy}

Prophylactic chemotherapy using methotrexate can cause leukoencephalopathy. The dose of intravenous (not intrathecal) MTX correlates with the incidence of leukoencephalopathy, regardless of history of radiation therapy. The prevalence of leukoencephalopathy is reported to be 


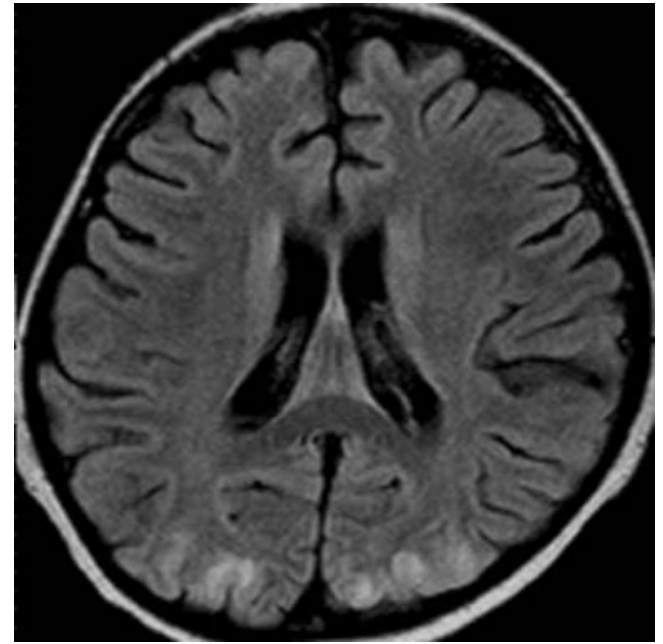

Fig. 7 A 7-year-old girl after BMT (day 62) for chronic EV virus infection and treated with cyclosporine A, presenting with a sudden seizure. Axial FLAIR MR image shows cortical-subcortical abnormal hyperintense lesions in the bilateral parieto-occipital lobes. These imaging appearances are typically consistent with PRES

from 16 to $69 \%$. Neurological symptoms may or may not be present, and the neurological outcome is good in many cases. Typically, patients have neurological symptoms when an injury occurs acutely. This is not the precursor of disseminated necrotizing leukoencephalopathy $[30,35,36]$.

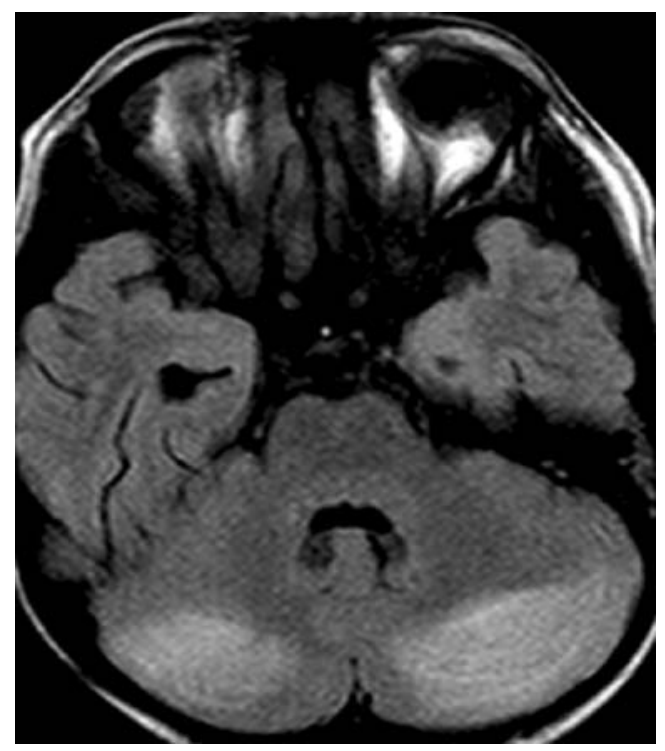

Fig. 8 A 5-year-old girl after BMT (day 25) because of Fanconi anemia who presented with a seizure. She was being treated with tacrolimus for GVHD. Axial FLAIR MR image shows pathy hyperintense lesions in the bilateral cerebellar hemisphere and left parieto-occipital lobe. The cortical-subcortical lesions predominantly in the parieto-occipital lobe are consistent with PRES. The cerebellar involvement as in this case is rarely seen
Imaging findings MRI is more sensitive than CT for detection of lesions. MRI shows bilateral high signal intensity, primarily in central and periventricular white matter on T2WI and FLAIR, with or without diffusion restriction (Fig. 9). Subcortical white matter is relatively spared. The corpus callosum, hippocampal commissure, and anterior commissure are most often spared. The lesions are transient, but may remain visible for a long time after the disappearance of clinical symptoms. If foci of contrast enhancement and of T1 and T2 shortening are seen, the development of diffuse necrotizing leukoencephalopathy should be considered (see next section) [17, 30].

\section{Disseminated necrotizing leukoencephalopathy (DNL)}

Diffuse white matter injury after intrathecal administration of methotrexate with or without cranial radiotherapy is a not infrequent CNS complication. Rapidly progressive deterioration occurs clinically, presenting with drowsiness, confusion, seizure, ataxia, and dementia resulting in poor neurological outcome. This form of leukoencephalopathy is differentiated from other forms, such as PRES and MTX-induced leukoencephalopathy, by the presence of more extensive areas of white matter necrosis in DNL [17, $36,37]$.

Imaging findings On MRI, progressive signal changes with mass effects in deep white matter represent diffuse

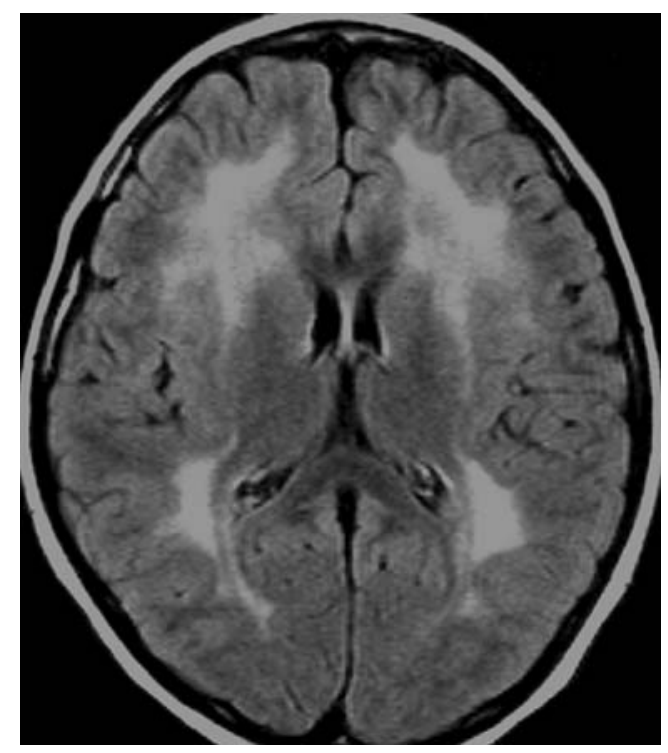

Fig. 9 An 8-year-old boy after BMT because of non-Hodgkin lymphoma. Before BMT, MTX/hydrocortisone therapy was performed. Axial FLAIR MR image demonstrates hyperintense changes in the bilateral deep white matter without T2 shortening foci. The cerebral atrophy is thought to be due to the chemotherapy 
necrotizing foci as $\mathrm{T} 1-$ and T2-shortening lesions (Fig. 10). Injury to the blood-brain barrier results in marked contrast enhancement with irregular ring-like shapes. Later, calcifications may be seen on CT if the patients survive [17, 36, 37].

\section{Metabolic disturbance following BMT}

Metabolic disturbances involving the CNS are less frequent than other CNS complications after BMT, but may be lifethreatening occasionally. They usually develop during the first to second phase after BMT and may result from renal failure, hepatic failure, electrolyte imbalance, and hypoxia [4]. Among the CNS complications following BMT, Wernicke's encephalopathy has been reported in $5.5 \%$ at autopsy [8]. Central pontine myelinolysis may also develop, but is very rare [39].

Children given BMT before puberty may later develop growth impairment caused by prophylactic chemotherapy with or without irradiation therapy. Injury to the hypothalamic-pituitary axis leads to inappropriately low production of growth hormone. Annual growth evaluation should be performed in all children after BMT. Less frequently, delayed puberty and deficiency of secondary sex characters can develop [1].

\section{Central pontine myelinolysis}

Central pontine myelinolysis is a rare CNS complication after BMT, being more common following liver transplantation (29\% at autopsy after liver transplantation)
[39]. It is a demyelinating disorder affecting mainly the central part of the pons, accompanied by extrapontine myelinolysis in some cases. Demyelination is thought to be related to cellular (and/or vasogenic) edema, caused by hyponatremia especially in the case of rapid correction or associated with hypoxia. Patients deteriorate subacutely (usually within a few days) to coma, quadriplegia, pseudobulbar palsy, and extrapyramidal motor symptoms $[17,30]$.

Imaging findings $\mathrm{CT}$ and MRI show a symmetrical lesion with a typical " $\omega$ " or "batwing" shape in the center in the pons (hypoattenuation on $\mathrm{CT}$ and $\mathrm{T} 2$-prolongation on MRI). MR images (especially T2WI) have higher sensitivity than CT. Corticospinal tracts may be spared. Restriction of diffusion occurs in the subacute phase (within a few days). Extrapontine involvement including the thalamus, basal ganglia, and cerebellum may be observed [30].

\section{Post-BMT carcinogenesis}

Post-transplantation lymphoproliferative disorder (PTLD), therapy-induced neoplasm, and CNS relapse can appear in the late phase after BMT. PTLD following BMT in children is a rare, but life-threatening complication. On the other hand, the overall risk of developing secondary brain tumors is significantly higher after BMT than in the general population. Radiation-induced telangiectases and secondary neoplasms such as glioblastoma multiforme and meningioma are well known. CNS relapse may also occur (Fig. 11).

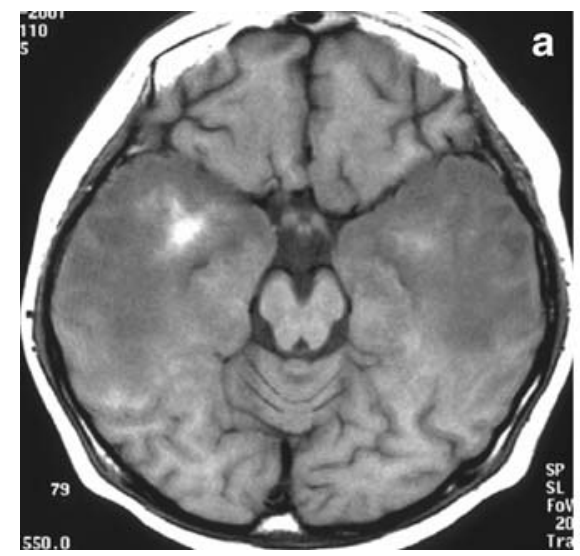

Fig. 10 A 16-year-old boy with ALL after prophylactic therapy using intrathecal high-dose MTX therapy before BMT. He presented with seizure, confusion, and drowsiness. a On axial T1-weighted MR image, irregular T1-shortening areas with widespread surrounding edema can be detected. b Axial T2-weighted image shows the

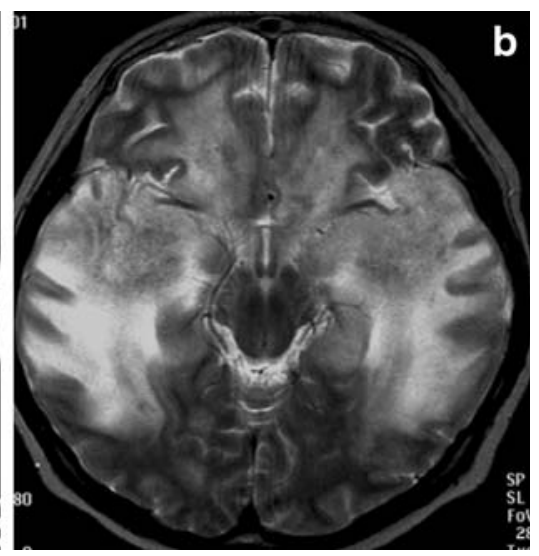

necrotizing foci as T2-shortening area with edema diagnosed as disseminated necrotizing leukoencephalopathy (DNL). His neurological symptoms deteriorated, and BMT had to be abandoned. He died 80 days after the neurological insult 
Fig. 11 A 23-year-old boy who had received BMT 1 year ago for non-Hodgkin lymphoma presented with right deviation of his tongue. a Post-contrast T1weighted coronal image shows an irregular mass-like lesion in the right skull base along to the hypoglossal canal with contrast enhancement. b A post-contrast T1-weighted sagittal image shows parietal skull involvement. Multiple vertebral involvements were also seen (not shown). Biopsy confirmed recurrence of non-Hodgkin lymphoma
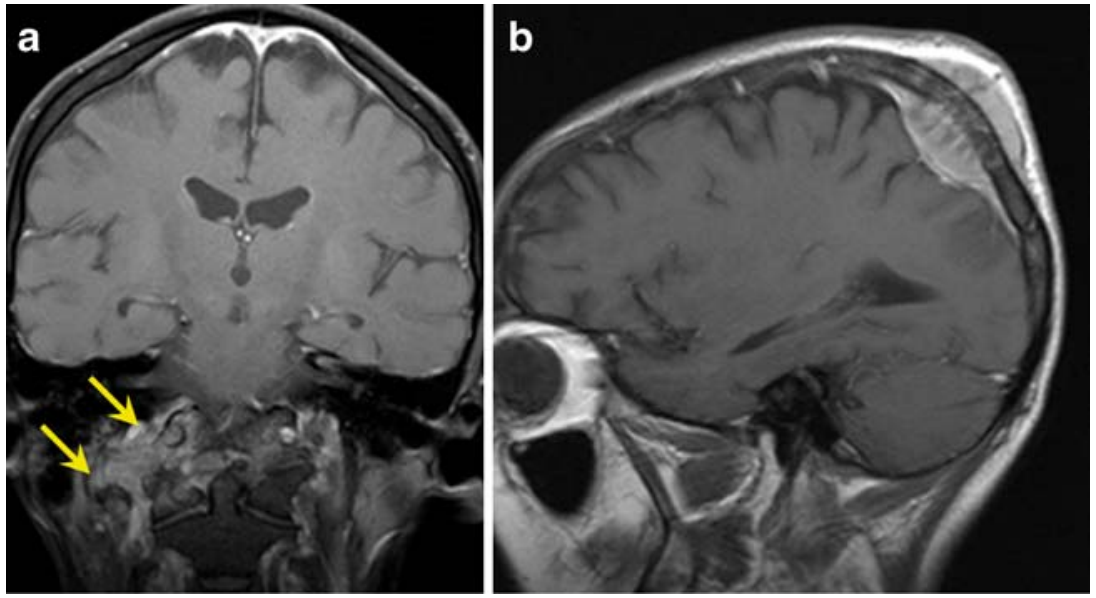

Post-transplantation lymphoproliferative disorder (PTLD)

Chronic immunosuppression leads to an unregulated expansion of lymphoid cells in patients after allogenic BMT [40-42]. Post-transplantation lymphoproliferative disorder (PTLD) represents a spectrum of unregulated lymphoid expansion that ranges from polyclonal B-cell proliferation to monoclonal malignant lymphoma. PTLD can occur from about 6 weeks to 7 years after transplantation. In most cases, the disorder results from Epstein-Barr virus (EBV)-induced $\mathrm{B}$ cell proliferation related to pharmacological suppression of the $\mathrm{T}$ cell system. Allograft-type BMT, Epstein-Barr virus infection, and immu- nosuppression are risk factors increasing the incidence of PTLD. The incidence of PTLD after BMT is reported to be $0.6-10 \%$, with low frequency in children after autologous BMT [40, 43, 44]. In children, brain involvement occurs less commonly, in 1 to $6 \%$ of cases among all kinds of organ transplantation (abdomen 64\%, thorax 50\%, head and neck $25 \%$ ), but CNS involvement is associated with poor prognosis [41].

Imaging findings $\mathrm{CT}$ and $\mathrm{MR}$ images are both useful modalities for detection of lesions. Diffuse cervical lymphadenopathies, orbital soft tissue mass, and sinonasal involvement can be observed. In the brain parenchyma, solitary periventricular-subcortical mass can be seen.
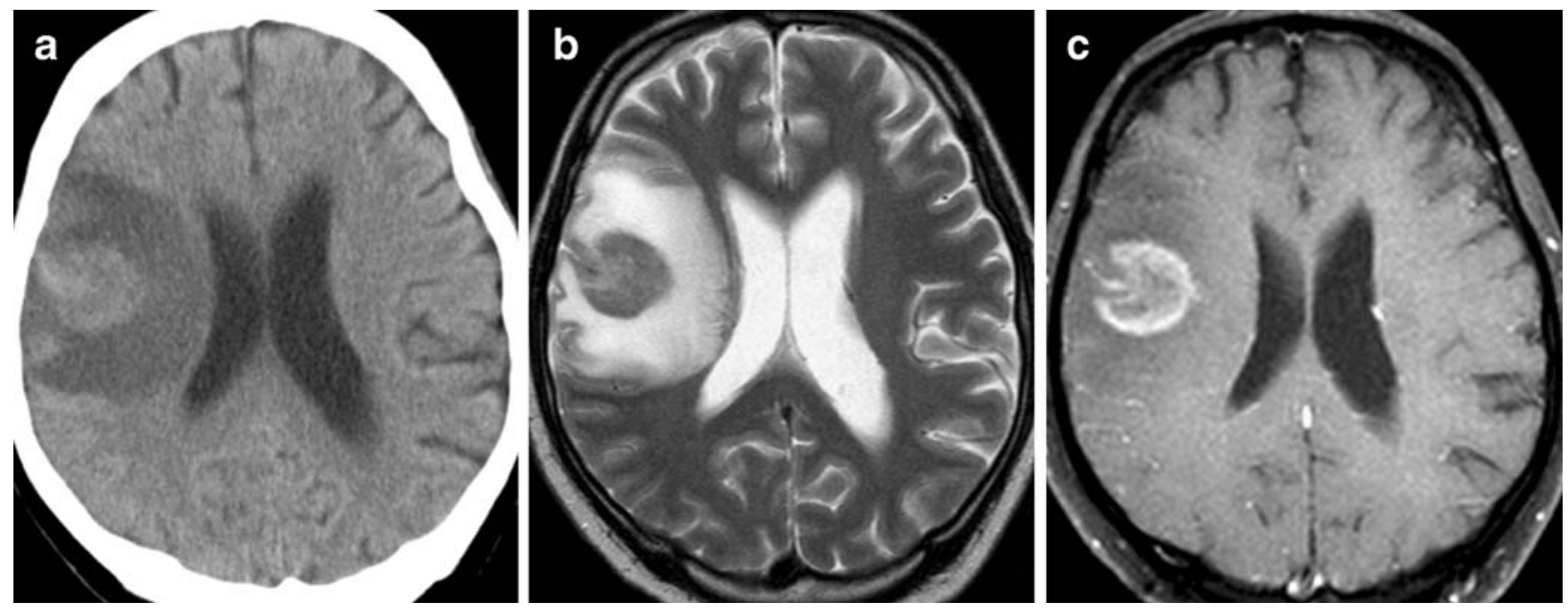

Fig. 12 A 32-year-old man after BMT 6 months ago because of ALL. He presented with left hemiparesis and fever. a On plain axial CT, a solitary subcortical high density lesion can be seen. b Axial T2- and T1- (not shown) weighted MR images depict the isointensity mass lesion in the right temporal lobe. Diffusion restriction of the lesion is seen on ADC map (not shown). c Postcontrast T1-weighted axial image reveals irregular contrast-enhanced mass. Initially, brain abscess was suspected, and drainage was performed; pathology showed this mass to be PTLD (courtesy of Dr. K. Ichihashi, Kurashiki Central Hospital) 
Intertumoral hemorrhage and necrosis are more frequently seen in PTLD than in primary CNS lymphoma. CT shows highly attenuated lesions reflecting hypercellularity or hemorrhage. MR images can reveal hemorrhage and necrosis in the mass lesion, with ring-like enhancement on administration of contrast material (Fig. 12) [41-42]. Since these imaging findings are often non-specific and the differentiation from the other disease entities is not straightforward, biopsy is usually required to help to confirm the diagnosis.

\section{Conclusion}

The frequency of various CNS complications after BMT differs according to the period after BMT. Except for the detection of hemorrhage or calcification, MRI is generally the optimal imaging investigation. In particular, T2WI and FLAIR images are useful because of their higher sensitivity for detecting white matter lesions compared with other sequences. DWI is also helpful for differentiation of abscess or ischemic lesion. It is essential that radiologists understand how imaging findings correlate with clinical features.

\section{References}

1. Kliegman RM, Jensen HB, Behman RE, Stanson BF (2004) Nelson textbook of Pediatrics. 18th ed.Saunders Elsevier, Philadelphia, 924-34:1104-7

2. Kato K, Harada M (2005) Present status in hematopoietic stem cell transplantation: peripheral blood stem cell transplantation. Nippon Naika Gakkai Zasshi 94:1281-1286

3. Harada M (2003) Hematopoietic stem cell transplantation therapy for leukemia. Nippon Naika Gakkai Zasshi 92:999-1006

4. Wiznitzer M, Packer RJ, August CS, Burkey ED (1984) Neurological complications of bone marrow transplantation in childhood. Ann Neurol 16:569576

5. Sostak P, Padovan CS, Yousry TA, Ledderose G, Kolb HJ, Staube A (2003) Prospective evaluation of neurological complications after allogenic bone marrow transplantation. Neurology 60:842-848

6. Antonini G, Ceschin V, Morino S et al (1998) Early neurologic complications following allogenic bone marrow transplant for leukemia: a prospective study. Neurology 50:1441-1445

7. Graus F, Saiz A, Sierra J et al (1996) Neurologic complications of autologous and allogenic bone marrow transplantation in patients in leukemia: a comparative study. Neurology 46:1004-1009
8. Bleggi-Torres LF, Medeiros BC, Werner B et al (2000) Neuropathological findings after bone marrow transplantation: an autopsy study of 180 cases. Bone Marrow Transplant 25:301-307

9. Levine DS, Navarro OM, Chaudry G, Doyle JJ, Blaser S (2007) Imaging the complications of bone marrow transplantation in children. Radiographics 27:307-325 DOI 10.1148/ rg. 272065088

10. Uckan D, Cetin M, Yigitkanli I et al (2005) Life-threatening neurological complications after bone marrow transplantation in children. Bone Marrow Transplant 35:71-76 DOI 10.1038/sj.bmt. 1704749

11. Dykewicz CA (2001) Summary of the Guidelines for preventing opportunistic infections among hematopoietic stem cell transplantat recipients. Clin Infect Dis 33:139-144 DOI 10.1086/321805

12. Sullivan KM, Dykewicz CA, Longworth DL et al (2001) Preventing opportunistic infections after hematopoietic stem cell transplantation: the Centers for Disease Control and Prevention, Infectious Diseases Society of America, and American Society for Blood and Marrow Transplantation Practice Guidelines and beyond. Hematology. Am Soc Hematol Educ Program 392-421

13. Coley SC, Jager HR, Szydlo RM et al (1999) CT and MRI manifestations of central nervous system infection following allogenic bone marrow transplantation. Clin Radiol 54:390-397 DOI 10.1053/crad.1999.0200
14. Guermazi A, Gluckman E, Tabti B, Myaux Y (2003) Invasive central nervous system aspergillosis in bone marrow transplantation recipients: an overview. Eur Radiol 13:377-388 DOI 10.1007/s00330-002-1480-5

15. Miaux Y, Williams M, Guermazi A et al (1995) MR of cerebral aspergillosis in patients who have had bone marrow transplantation. Am J Neuroradiol 16:555-562

16. Yamada K, Shrier DA, Rubio A et al (2002) Imaging findings in intracranial aspergillosis. Acad Radiol 9:163-171

17. Barkovich AJ (2005) Pediatric Neuroimaging. 4th. Lippincott Williams \& Wilkins, Philadelphia, pp 843-844

18. Yoshikawa T (2003) Human herpesvirus6 and -7 infections in transplantation. Pediatr Transplantation 7:11-17 DOI 10.1034/j.1399-3046.2003.02094.x

19. Gorniak RJT, Young GS, Wiese DE, Marty FM, Schwarz RB (2006) MR imaging of human herpesvirus-6-associated encephalitis in 4 patients with anterograde amnesia after allogenic hematopoietic stem cell transplantation. Am J Neuroradiol 27:887-891

20. Urbach H, Soeder BM, Jeub M, Klockgether T, Meyer B, Bien CG (2006) Serial MR of limbic encephalitis. Neuroradiology 48:380-386 DOI 10.1007/s00234-006-0069-0

21. Baskin HJ, Hedlund G (2007) Neuroimaging of herpes virus infections in children. Pediatr Radiol 37:949-963 DOI 10.1007/s00247-007-0506-1 
22. Fukui MB, Williams RL, Mudigonda S (2001) CT and MR imaging features of pyogenic ventriculitis. AJNR 22:15101516

23. Pezzullo JA, Tung GA, Mudigonda S, Rogg JM, Jeffrey MR (2003) Diffusion-weighted MR imaging of pyogenic ventriculitis. AJR 180:71-75

24. Rana S, Albayram S, Lin DDM, Yousem DM (2002) Diffusionweighted imaging and apparent diffusion coefficient maps in a case of intracerebral abscess with ventricular extension. AJNR 23:109-112

25. Han KT, Choi DS, Ryoo JW et al (2007) Diffusion-weighted MR imaging of pyogenic intraventricular empyema. Neuroradiology 49:813-818 DOI 10.1007/s00234-007-0264-7

26. Osorio S, Camara R, Golbano N et al (2002) Progressive multifocal leukoencephalopathy after stem cell transplantation, unsuccessfully treated with cidofovir. Bone Marrow Transplant 30:963-966 DOI 10.1038/sj. bmt. 1703704

27. Unal S, Varan A, Yalcin B, Buyukpamukcm M, Gurgey A (2005) Evaluation of thrombotic children with malignancy. Ann Hematol 84:395-399 DOI 10.1007/s00277-005-1004-x

28. Mitchell LG, Andrew M, Hanna K et al (2003) A prospective cohort study determining the prevalence of thrombotic events in children with acute lymphoblastic leukemia and a central venous line who are treated with Lasparaginase: results of the Prophylactic Antithrombin Replacement in Kids with Acute Lymphoblastic Leukemia Treated with Asparaginase (PARKAA) study. Cancer 15:508-516 DOI 10.1002/cncr. 11042
29. Ma M, Barnes G, Pulliam J, Jezek D, Baumann RJ, Berger JR (2002) CNS angitis in graft vs host disease. Neurology 59:1994-1997

30. Knaap MS, Valk J (2005) Magnetic resonance of myelination and myelin disorders. 3rd ed. Springer, Berlin, Heidelberg, 679-689, 808-817

31. Schwarz RB, Bravo SM, Klufas RA et al (1995) Cyclosporine neurotoxicity and its relationship to hypertensive encephalopathy: CT and MR findings in 16 cases. AJR 165:627-631

32. Mckinney AM, Short J, Truwit CL et al (2007) Posterior reversible encephalopathy syndrome: incidence of atypical regions of involvement and imaging findings. AJR 189:904-912 DOI 10.2214/AJR.07.2024

33. Shimono T, Miki Y, Toyoda $\mathrm{H}$ et al (2003) MR imaging with quantitative diffusion mapping of tacrolimus-induced neurotoxicity in organ transplant patients. Eur Radiol 13:986-993 DOI 10.1007/s00330-002-1594-9

34. Trullemans F, Grignard F, Camp B, Schots R (2001) Clinical findings and magnetic resonance imaging in severe cyclosporine-related neurotoxicity after allogenic bone marrow transplantation. Eur J Haematol 67:94-99 DOI 10.1034/j.1600-0609.2001.t011-00440.x

35. Pande AR, Ando K, Ishikura R et al (2006) Clinicoradiological factors influencing the reversibility of posterior reversible encephalopathy syndrome: a multicenter study. Radiat Med 24:659668 DOI 10.1007/s11604-006-0086-2

36. Reddick WE, Glass JO, Helton KJ et al (2005) Prevalence of leukoencephalopathy in children treated for acute lymphoblastic leukemia with high-dose methotrexate. AJNR 26:1263-1269

37. Reddick WE, Glass JO, Helton KJ et al (2005) A quantitative MR imaging assessment of leukoencephalopathy in children treated for acute lymphoblastic leukemia without irradiation. AJNR 26:2371-2377
38. Pande AR, Ando K, Ishikura $\mathrm{R}$ et al (2006) Disseminating necrotizing leukoencephalopathy following chemoradiation therapy for acute lymphoblastic leukemia. Radiat Med 24:515-519 DOI 10.1007/s11604-006-0059-5

39. Fraser C, Charnas L, Orchard P (2005) Central pontine myelinolysis following bone marrow transplantation complicated by severe hepatic veno-occlusive disease. Bone Marrow Transplant 36:733-734 DOI 10.1038/sj. bmt. 1705115

40. Lones MA, Said JW, Shintaku IP, Kirov I, Neudorf S (2000) Post-transplant lymphoproliferative disorder after autologous peripheral stem cell transplantation in a pediatric patient. Bone Marrow Transplant 26:1021-1024

41. Pickhardt PJ, Siegel MJ, Hayashi RJ, Kelly M (2000) Posttransplantation lymphoproliferative disorder in children: clinical, histopathologic, and imaging features. Radiology 217:16-25

42. Scarsbrook AF, Warakaulle DR, Dattani M, Traill Z (2005) Posttransplantation lymphoproliferative disorder: the spectrum of imaging appearances. Clin Radiol 60:47-55 DOI 10.1016/j.crad.2004.08.016

43. Gross TG, Steinbuch M, DeFor T et al (1999) B cell lymphoproliferative disorders following hematopoietic stem cell transplantation: risk factors, treatment and outcome. Bone Marrow Transplant 23:251-258

44. Kasow KA, Leung W, Horwitz EM, Woodand P, Handgretinger R, Hale GA (2007) EBV lymphoproliferative disease of host origin after haploidentical stem cell transplantation. Pediatr Blood Cancer 49:869-872 DOI 10.1002/ pbc. 20710 